\title{
Diagnostic evaluation of magnetization transfer and diffusion kurtosis imaging for prostate cancer detection in a re-biopsy population
}

\author{
Tristan Barrett ${ }^{1,2,3} \cdot$ Mary McLean $^{4} \cdot$ Andrew N. Priest $^{2} \cdot$ Edward M. Lawrence $^{1,5}$. \\ Andrew J. Patterson ${ }^{2}$ Brendan C. Koo ${ }^{2,3}$ • Ilse Patterson ${ }^{2}$. Anne Y. Warren ${ }^{3,6}$ • \\ Andrew Doble ${ }^{3,7}$ - Vincent J. Gnanapragasam ${ }^{3,7}$ - Christof Kastner ${ }^{3,7}$. \\ Ferdia A. Gallagher ${ }^{1,2}$
}

Received: 23 June 2017 /Revised: 23 October 2017 / Accepted: 3 November 2017 /Published online: 8 December 2017

(C) The Author(s) 2017. This article is an open access publication

\begin{abstract}
Objective To evaluate diffusion kurtosis imaging (DKI) and magnetisation transfer imaging (MTI) compared to standard MRI for prostate cancer assessment in a re-biopsy population. Methods Thirty-patients were imaged at $3 \mathrm{~T}$ including DKI $\left(\mathrm{K}_{\text {app }}\right.$ and $\mathrm{D}_{\text {app }}$ ) with b-values $150 / 450 / 800 / 1150 / 1500 \mathrm{~s} / \mathrm{mm}^{2}$ and MTI performed with and without MT saturation. Patients underwent transperineal biopsy based on prospectively defined MRI targets. Receiver-operating characteristic (ROC)
\end{abstract}

Electronic supplementary material The online version of this article (https://doi.org/10.1007/s00330-017-5169-1) contains supplementary material, which is available to authorized users.

Tristan Barrett

tb507@medschl.cam.ac.uk

Department of Radiology, University of Cambridge, Cambridge, UK

2 Department of Radiology, Addenbrooke's Hospital, Cambridge CB2 OQQ, UK

3 CamPARI Clinic, Addenbrooke's Hospital and University of Cambridge, Cambridge, UK

4 CRUK Cambridge Institute, Cambridge, UK

5 Present address: Department of Radiology, Memorial Sloan Kettering Cancer Center, 1275 York Ave, New York, NY, USA

6 Department of Histopathology, Addenbrooke's Hospital and University of Cambridge, Cambridge, UK

7 Department of Urology, Addenbrooke's Hospital and University of Cambridge, Cambridge, UK analyses assessed the parameters and Wilcoxon-signed ranked test assessed relationships between metrics.

Results Twenty patients had $\geq 1$ core positive for cancer in a total of $26 \mathrm{MRI}$ targets (Gleason $3+3$ in $8,3+4$ in $12, \geq 4+3$ in 6): 13 peripheral (PZ) and 13 transition zone (TZ). The apparent diffusion coefficient (ADC) and $\mathrm{D}_{\text {app }}$ were significantly lower and the $\mathrm{K}_{\text {app }}$ and MT ratio (MTR) significantly higher in tumour versus benign tissue (all $p \leq 0.005$ ); ROC values 0.767-1.000. Normal TZ had: lower ADC and $\mathrm{D}_{\text {app }}$ and higher $\mathrm{K}_{\text {app }}$ and MTR compared to normal PZ. MTR showed a moderate correlation to $\mathrm{K}_{\text {app }}(r=0.570)$ and $\mathrm{D}_{\text {app }}(r=-0.537)$ in normal tissue but a poor correlation in tumours. No parameter separated lowgrade (Gleason $3+3)$ from high-grade $(\geq 3+4)$ disease for either PZ $(p=0.414-0.825)$ or TZ $(p=0.148-0.825)$.

Conclusion ADC, $\mathrm{D}_{\text {app }}, \mathrm{K}_{\mathrm{app}}$ and MTR all distinguished benign tissue from tumour, but none reliably differentiated lowfrom high-grade disease.

Key Points

- MTR was significantly higher in PZ and TZ tumours versus normal tissue

- $K_{\text {app }}$ was significantly lower and $D_{\text {app }}$ higher for PZ and TZ tumours

- There was no incremental value for DKI/MTI over monoexponential ADC parameters

- No parameter could consistently differentiate low-grade (Gleason 3+3) from high-grade $(\geq 3+4)$ disease

- Divergent MTR/DKI values in TZ tumours suggests they offer different functional information

Keywords MRI · Prostate · Diffusion kurtosis imaging · Magnetisation transfer imaging · Diffusion-weighted imaging 


\section{Introduction}

Prostate cancer is the second leading cause of cancer in men, accounting for around $20 \%$ of male cancer diagnoses [1]. The diagnosis of prostate cancer is primarily based on transrectal ultrasound (TRUS)-guided biopsies. However, this non-targeted sampling approach means around a third of tumours will be under-graded and half missed altogether $[2,3]$. In patients with an initial negative biopsy, but continued clinical suspicion of prostate cancer or suspicion of an under-sampled lesion, national guidelines in the UK recommend further assessment to exclude or confirm the presence of aggressive tumour [4]. Multiparametric MRI (mpMRI) is used to guide rebiopsy in such patients and has been shown to outperform systematic TRUS biopsy for lesion detection [5]. However, studies report considerable variation in MRI performance, with sensitivity and specificity ranging from $73-100 \%$ and $8-100 \%$, respectively $[6,7]$, depending on composition of the study population, radiologist experience, technical issues, and the gold standard employed. Furthermore, specificity drops from $80 \%$ to $47 \%$ when including indeterminate lesions, i.e. Prostate ImagingReporting and Data System (PI-RADS) score 3 lesions in addition to score 4-5 [6,7]. This highlights the need for improvement of existing sequences, or use of additional functional sequences, with the most recent version of the PI-RADS guidelines strongly supporting the continued development of further novel MRI sequences [8]. Here we study two imaging techniques in prostate cancer-diffusion kurtosis imaging (DKI) and magnetisation transfer imaging (MTI). DKI is a novel technique for studying the heterogeneity of water diffusion that has recently been applied to the prostate, and MTI is a more established technique for probing macromolecules in the microenvironment, but there has been limited work using this method in prostate cancer; neither approach requires additional administration of exogenous contrast agents and both could be translated into routine clinical practice if effective.

DKI is a form of diffusion-weighted imaging that quantifies the degree to which water diffusion in tissues differs from what would be expected under a normal (Gaussian) distribution of displacements, and from the corresponding monoexponential decay of signal with increasing b-value [9], deriving two parameters. The apparent diffusivity $\mathrm{D}_{\text {app }}$ quantifies the exponential component of signal decay and is similar to the apparent diffusion coefficient (ADC) in the standard mono-exponential model. The apparent kurtosis $\mathrm{K}_{\mathrm{app}}$ measures the first-order deviation from mono-exponential decay and is thus a simple measurement of the deviation from a Gaussian distribution of displacements. Theoretically, kurtosis values may quantify the variability in tissue structure within the region of interest, providing a measurement of intra-voxel tissue heterogeneity, and may be useful for assessing structural abnormalities in pathologic regions. Previous studies have suggested a possible correlation between $\mathrm{K}_{\mathrm{app}}$ and prostate cancer aggressiveness [10,11], although a recent large retrospective study showed that despite DKI itself performing well, no added value was observed over standard DWI sequences [12].

MTI detects the interaction between free and bound water molecules. The "free" pool consists of relatively mobile protons and provides the majority of the visible MR signal. The "bound" pool incorporates the "hydration layer" of water molecules bound to the surface of macromolecules. The protons associated with macromolecules are relatively immobile, with decay times being too rapid to detect an MR signal [13], however, the hydration layer is able to interact with the "free" pool and can modulate its relaxation properties and affect the measurable MR signal. This effect is exploited in MTI, where an off-resonance radiofrequency pulse saturates the nuclear magnetisation in the hydration layer; this in turn exchanges with the free pool of protons and thus reduces the MR signal. The MT ratio (MTR) is a simple derived metric and is therefore a measure of the structural integrity of tissues and can probe the microstructural changes induced by pathological processes [14]. MTR has been shown to change in malignancy because of changes in cell number and size as well as changes in cell membrane structures and extracellular space content, which may help differentiate primary for secondary brain tumours $[15,16]$. Preliminary work in prostate cancer has demonstrated a higher MTR within the peripheral zone of patients with prostate cancer compared to normal controls $[17,18]$.

Advanced functional imaging with DKI and MTI may provide insight into the tissue structure and the complicated micro-environment of prostate tumours. We therefore aimed to evaluate whether the addition of these two novel MR quantification parameters to standard MRI sequences could aid prostate cancer detection in a transperineal re-biopsy population.

\section{Methods}

\section{Patient population}

Thirty-patients with a clinical suspicion of undiagnosed prostate cancer were prospectively enrolled into this local institutional review board-approved (CUH/13/EE/0100) single-centre study between November 2013 and June 2016, with all subjects signing written informed consent. Inclusion criteria included prior negative biopsy with a suspicious lesion on MRI $(n=24)$ or prior diagnosis of low-grade prostate cancer and an MRI suspicious lesion 
in a remote gland location $(n=6)$. Patients subsequently underwent MR-TRUS fusion template transperineal biopsy, including target cores from MRI suspicious lesion/s.

\section{MR Imaging}

All patients underwent 3-T MRI (MR 750, GE-Healthcare, WI, USA) using a 32-channel phased-array coil. The protocol included multiplanar T2-weighted fast recovery fast spin-echo (FSE) images of the prostate and axial T1weighted FSE images of the pelvis. Standard clinical axial DWI was performed using a single spin-echo echo-planar imaging (EPI) pulse sequence with b-values of 150/1000/ $1400 \mathrm{~s} / \mathrm{mm}^{2}$, with automated ADC maps. DCE was performed as a 3D fast-spoiled gradient echo sequence; 85 100 dynamic phases were acquired with temporal resolution $7 \mathrm{~s}$; bolus gadobutrol (Schering AG) was injected intravenously via a power injector (rate $3 \mathrm{ml} / \mathrm{s}$, dose $0.1 \mathrm{mmol} / \mathrm{kg}$ ) followed by $25 \mathrm{ml}$ saline flush; total scan duration was $10 \mathrm{~min}$.

DKI was performed as a single-shot dual-spin-echo EPI pulse sequence, including five different $b$-values and a 'noise-only' image set with no RF pulses but otherwise identical acquisition parameters. MTI volumes covering the prostate were acquired with two 3D spoiled gradient recalled-echo acquisitions with and without an MT saturation pulse with slice thickness matched to T2-axial images.
The saturation pulse consisted of a $400^{\circ}$ Fermi shape pulse of 10 -ms duration and $800 \mathrm{~Hz}$ bandwidth at $2.5 \mathrm{kHz}$ offresonance frequency (Table 1).

\section{MRI-Guided Biopsy}

The MRIs were prospectively interpreted by one of two uroradiologists with $>4$ years' prostate MRI reporting experience. Images were analysed according to PI-RADS version 1 criteria [19] prior to February $2015(n=13)$ and subsequently using criteria described in PI-RADS version $2(n=17)$ [4]. In all cases, interpretation was based on a Likert scale: 1 , no suspicious area; 2 , cancer unlikely; 3 , indeterminate; 4 , cancer likely; 5 , cancer highly likely [19-22]. All studies were reviewed in a multidisciplinary team environment, which included radiologists, urologists, and oncologists, with all the clinical information available prior to the decision to undertake a biopsy. A positive lesion was defined as a Likert score $\geq$ 3. DKI and MTR values did not inform biopsy decision-making. The Biopsee ${ }^{\mathrm{TM}}$ MRI/TRUS-fusion biopsy system v.1 or v.2 (Medcom, Darmstadt, Germany) was used for all biopsies. A transrectal FlexFocus ${ }^{\mathrm{TM}}$ (BK-Medical, MA, USA) ultrasound probe was sited; the biplanar probe is fixed on a stepping unit and sagittal prostate images aligned with MRI using fusion software. Targets were prospectively drawn using $\mathrm{T} 2 \mathrm{~W}$ as primary and ADC as secondary source images. All patients underwent systematic transperineal biopsies
Table 1. Sequences in MRI protocol

\begin{tabular}{|c|c|c|c|c|}
\hline Parameter & $\begin{array}{l}\text { Axial T2 2D } \\
\text { FSE }\end{array}$ & DWI & DKI & MTI \\
\hline $\mathrm{TR}(\mathrm{ms})$ & $3000-5000$ & 4000 & 6000 & 24 \\
\hline $\mathrm{TE}(\mathrm{ms})$ & $99-106$ & $70-75$ & 94 & $2.4 / 4.8$ \\
\hline Averages & 3 & 8 & 6 & 1 \\
\hline $\begin{array}{l}\text { Section thickness } \\
\quad(\mathrm{mm})\end{array}$ & 3 & 4 & 3.6 & 4 \\
\hline Section gap (mm) & 1 & 0 & 0.4 & 0 \\
\hline FOV (mm) & $220 \times 220$ & $280 \times 280$ & $280 \times 280$ & $220 \times 220$ \\
\hline Matrix & $384 \times 288$ & $128 \times 128$ & $128 \times 96$ & $192 \times 160$ \\
\hline Resolution ( $\left.\mathrm{mm}^{2}\right)$ & $0.6 \times 0.8$ & $2.2 \times 2.2$ & $2.2 \times 2.9$ & $1.15 \times 1.38$ \\
\hline $\begin{array}{l}\text { Receiver bandwidth } \\
\quad( \pm \mathrm{kHz})\end{array}$ & 50 & 111 & 111 & 31 \\
\hline ASSET factor & No ASSET & 2 & 2 & $\begin{array}{l}\text { No } \\
\text { ASSET }\end{array}$ \\
\hline Time & $4: 39$ & $2: 58$ & $11: 30$ & $2: 50$ \\
\hline Other & $\begin{array}{l}\text { ETL } 16 \\
\text { No phase } \\
\text { wrap }\end{array}$ & $\begin{array}{l}\text { b-values } 0,1400 \\
\mathrm{~s} / \mathrm{mm}^{2}\end{array}$ & $\begin{array}{l}\text { b-values } 150,450,800,1150 \text {, } \\
\quad 1500 \mathrm{~s} / \mathrm{mm}^{2}\end{array}$ & $\underset{5^{\circ}}{\text { Flip angle }}$ \\
\hline
\end{tabular}

FSE, fast spin echo; EPI, echo planar imaging; DWI, diffusion-weighted imaging; FOV, field of view; ETL, echo train length; DKI, diffusion kurtosis imaging; MTI, magnetisation transfer imaging 
according to the Ginsburg protocol, using a spring-loaded 18$\mathrm{G}$ biopsy needle via a brachytherapy template grid [23]. In all cases two biopsy cores were taken from each lesion(s) first, with 24 background systematic biopsies subsequently acquired. All procedures were performed by one of three urologists with several years' experience of transperineal biopsy. All biopsies were Gleason-graded by a specialist uropathologist, following ISUP 2005 recommendations [24].

\section{Image Analysis}

Prospectively defined target outlines are stored on the local PACS system during the clinical workflow. A fellowshiptrained uroradiologist with 7-years' clinical prostate MR reporting experience reviewed these original outlines and redrew freehand ROIs on T2-weighted axial images, avoiding inclusion of the urethra or extra-prostatic tissue where relevant, using in-house software programmed with MATLAB (version 2016a; MathWorks, Natick, MA, USA). Additional ROIs were drawn in regions of the biopsy-benign peripheral zone (PZ) and transition zone (TZ) from the side contralateral to the target over three consecutive slices, with a minimum volume of $0.5 \mathrm{~cm}^{3}$. ROIs were secondarily transposed onto ADC, DKI, and MTR maps; in cases with significant distortion on any of the sequences, ROIs were adjusted to allow for this, using the targets and prostate outline on T2-weighted images for reference. DKI ( $\mathrm{D}_{\text {app }}$ and $\left.\mathrm{K}_{\mathrm{app}}\right)$ and MTR parameter maps were calculated offline using custom software written in Matlab. For DKI, the noise-only images were used to reduce and partially compensate for noise-floor bias, which could otherwise artificially enhance the kurtosis measurement [10]. A small proportion of DKI fit-failure pixels were excluded. The mean ROI values of ADC, $\mathrm{D}_{\mathrm{app}}$, and $\mathrm{K}_{\mathrm{app}}$ and MTR maps were used for analysis.

\section{Statistical analysis}

Medians and ranges were used to summarise continuous variables. Wilcoxon signed-rank test assessed the relationships between parameters, benign versus tumour tissue, and tumour grades in both the PZ and TZ. Receiver-operating characteristic (ROC) analyses were used to assess the diagnostic utility of metrics for detecting tumour and for discriminating Gleason grade. An optimal threshold was determined for each metric for discriminating tumour from benign tissue. Pearson's correlation assessed the relationship between the metrics. All statistical analysis was performed in $\mathrm{R}$ (version 3.1.1, The R-Foundation, Austria); $p<0.05$ was considered statistically significant.

\section{Results}

Thirty patients were included, with a median age of 65.5 years (range 50-76 years) and a median PSA of $7.67 \mathrm{ng} / \mathrm{ml}$ (IQR: $6.12-11.98 \mathrm{ng} / \mathrm{ml})$. The median time from MRI to biopsy was 26 days (IQR: 7.25-48.25 days). Twenty-six patients had a least one previous biopsy, with the interval from the most recent biopsy to MRI being at least 3 months (median 13, range 3-114, IQR 4-26 months).

Prostate cancer was detected in 24/30 patients including targeted and background cores and in 20 patients within at least one target core. In the four patients with only positive background cores, all had a Gleason score (GS) $3+3$ in 1-5\% of one core only. The remaining 20 patients had 26 separate MRI lesions with positive target cores (4 patients with 2 targets, 1 with 3), of which 13 were in the peripheral zone (PZ) and 13 in the transition zone (TZ) (Fig. 1). The final pathology of the 26 targets was GS $3+3(n=8), 3+4(12), 3+5(1), 4+3$
Fig. 1. Flow chart of biopsy outcomes

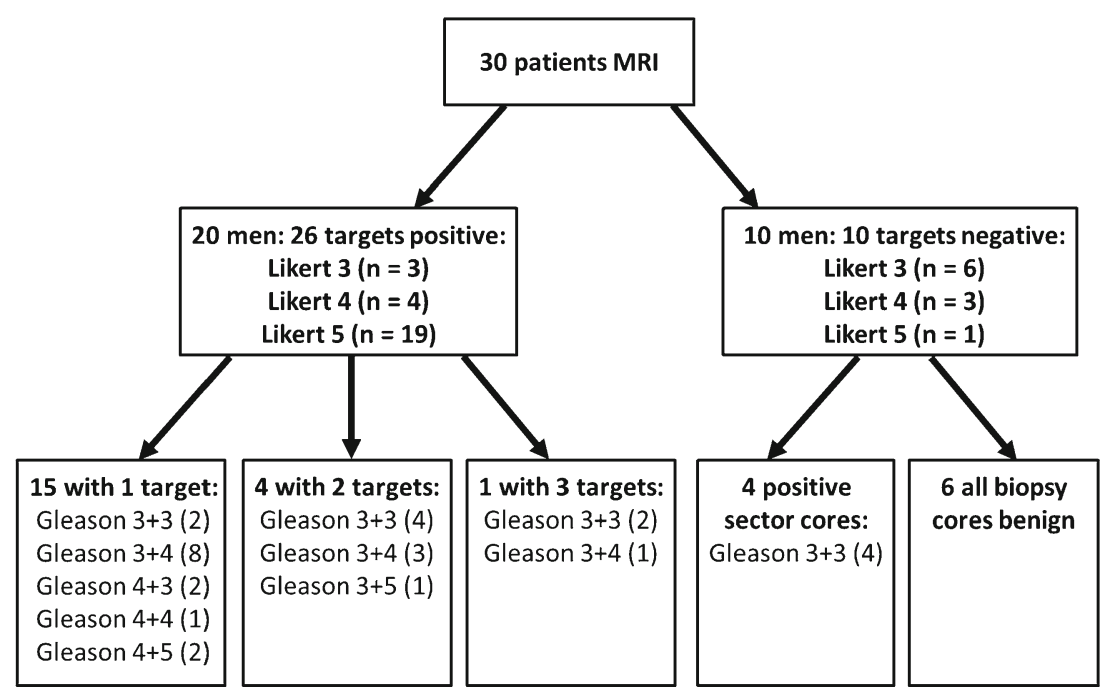


Table 2. Target biopsy outcomes for MRI-defined lesions and contralateral benign tissue

\begin{tabular}{|c|c|c|c|c|c|}
\hline Parameter & Normal tissue & Tumour target & Cystic BPH & $\begin{array}{l}p \text {-value(normal vs. } \\
\text { tumour) }\end{array}$ & $\begin{array}{l}p \text {-value(normal vs. cystic } \\
\text { BPH) }\end{array}$ \\
\hline \multicolumn{6}{|c|}{$A D C\left(\times 10^{-3} \mathrm{~mm}^{2} \mathrm{~s}^{-1}\right)$} \\
\hline $\begin{array}{l}\text { Peripheral } \\
\text { zone }\end{array}$ & $1.579[1.363-1.794]$ & $0.902[0.869-0.974]$ & N/A & $<0.001 *$ & N/A \\
\hline $\begin{array}{l}\text { Transition } \\
\text { zone }\end{array}$ & $1.270[1.244-1.355]$ & $0.845[0.813-0.885]$ & $1.955[1.669-2.031]$ & $<0.001 *$ & $<0.001$ \\
\hline \multicolumn{6}{|c|}{$D_{a p p}\left(\times 10^{-3} \mathrm{~mm}^{2} \mathrm{~s}^{-1}\right)$} \\
\hline $\begin{array}{l}\text { Peripheral } \\
\text { zone }\end{array}$ & $2.221[2.039-2.446]$ & $1.167[0.910-1.366]$ & N/A & $<0.001 *$ & N/A \\
\hline $\begin{array}{l}\text { Transition } \\
\text { zone }\end{array}$ & $1.808[1.712-1.895]$ & $0.951[0.853-1.062]$ & $2.152[2.113-2.258]$ & $<0.001 *$ & $<0.001 *$ \\
\hline \multicolumn{6}{|l|}{$K_{\text {app }}$ (unitless) } \\
\hline $\begin{array}{l}\text { Peripheral } \\
\text { zone }\end{array}$ & $0.507[0.426-0.550]$ & $0.716[0.621-0.869]$ & N/A & $0.004 *$ & N/A \\
\hline $\begin{array}{l}\text { Transition } \\
\text { zone }\end{array}$ & $0.615[0.548-0.653]$ & $0.871[0.663-0.1034]$ & $0.374[0.303-0.501]$ & $0.004 *$ & $0.001 *$ \\
\hline \multicolumn{6}{|l|}{$\operatorname{MTR}(\%)$} \\
\hline $\begin{array}{l}\text { Peripheral } \\
\text { zone }\end{array}$ & $20.0[15.5-22.9]$ & $25.0[24.0-28.1]$ & N/A & $<0.001 *$ & N/A \\
\hline $\begin{array}{l}\text { Transition } \\
\text { zone }\end{array}$ & $26.9[23.5-28.0]$ & $30.0[28.2-32.0]$ & $15.2[6.9-21.3]$ & $0.005 *$ & $<0.001 *$ \\
\hline
\end{tabular}

Mean values listed, interquartile range in parentheses; ${ }^{*} p<0.05$. BPH, benign prostatic hyperplasia

(2), and $\geq 4+4$ (3) (supplemental Table 1). The mean size for these 26 targets was $1.16 \mathrm{~cm}^{2}$ (range $0.22-4.71 \mathrm{~cm}^{2}$ ).
Mean $\mathrm{ADC}$ and $\mathrm{D}_{\text {app }}$ values for tumours in both the $\mathrm{PZ}$ and $\mathrm{TZ}$ were significantly lower than comparative normal PZ and
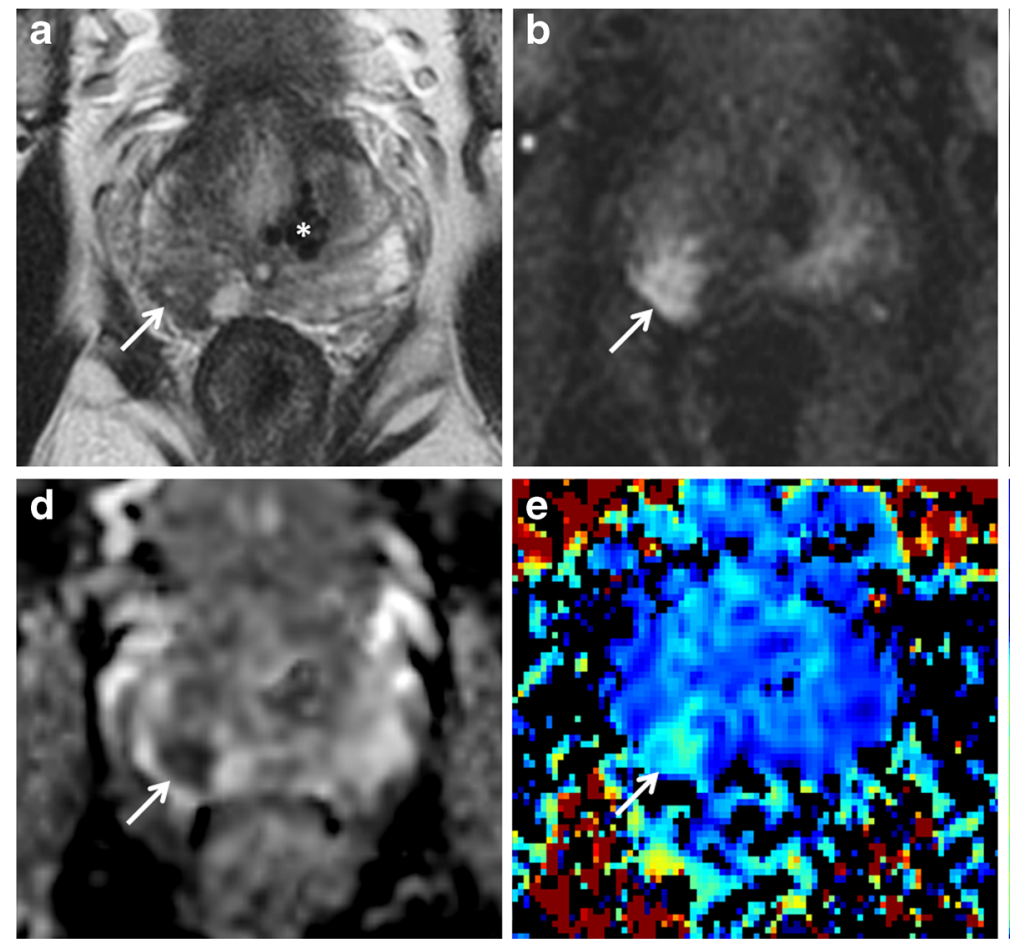

Target prospectively drawn in the right base PZ posteriorly (arrows). Note calcification in the left base $\mathrm{TZ}(*$ in a) with corresponding artefact on all other sequences. Target biopsy: $3+4=7$ (35\% pattern 4$)$ in $60 \%$ cores
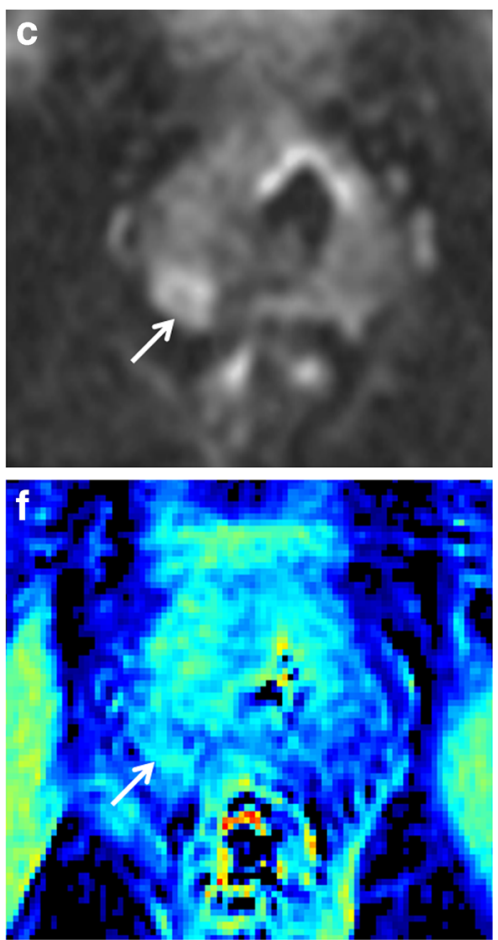

Fig. 2. Peripheral zone target. A 64-year-old male with a PSA of $6.5 \mathrm{ng}$ $\mathrm{ml}$. Top row: clinical mpMRI sequences: $\mathrm{T} 2 \mathrm{w}$ image (a), early time point DCE image (b), and $\mathrm{b}=1400 \mathrm{~s} / \mathrm{mm}^{2}$ diffusion-weighted image (c). Bottom row: assessed sequences: ADC map (d), $\mathrm{K}_{\text {app }}(\mathbf{e})$, and MTR (f). 

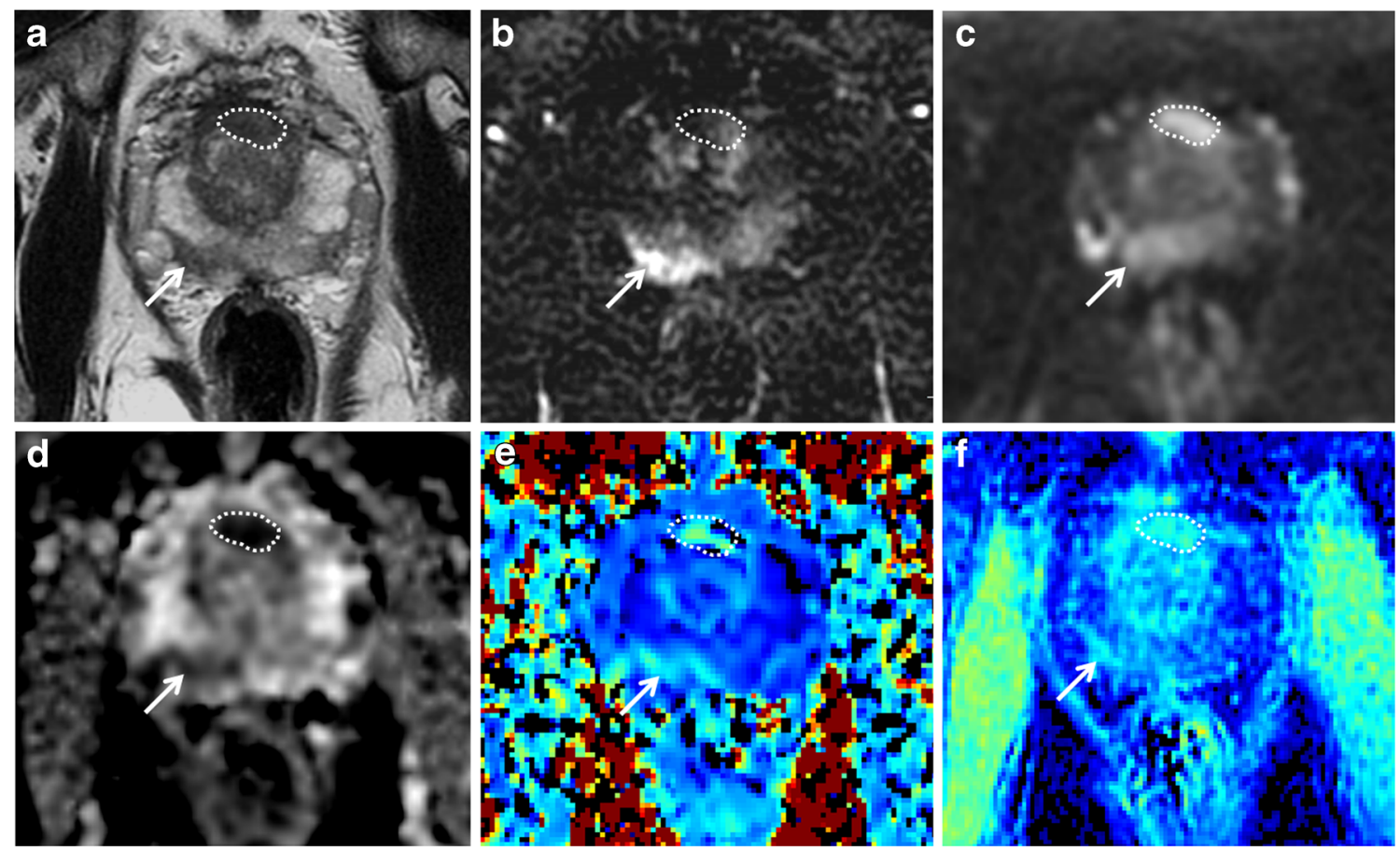

Fig. 3. Peripheral and transition zone target. A 69 -year-old male with PSA of $12.31 \mathrm{ng} / \mathrm{ml}$. Top row: clinical mpMRI sequences: T2w image (a), early time point DCE image (b), and $\mathrm{b}=1400 \mathrm{~s} / \mathrm{mm}^{2}$ diffusionweighted image (c). Bottom row: assessed sequences: ADC map (d), $\mathrm{K}_{\text {app }}(\mathbf{e})$, and MTR (f). Targets prospectively drawn in the anterior mid

TZ tissue (all $P$-values $<0.001$ ) (Table 2). Conversely, mean $\mathrm{K}_{\mathrm{app}}$ and MTR were significantly higher for PZ tumour and TZ tumour compared to normal PZ and normal TZ $(P \leq$ 0.005). Normal TZ had lower ADC and $\mathrm{D}_{\text {app }}$ and higher $\mathrm{K}_{\mathrm{app}}$ and MTR than normal PZ tissue, with cystic BPH demonstrating higher ADC and $\mathrm{D}_{\text {app }}$ and lower $\mathrm{K}_{\text {app }}$ and MTR compared to normal TZ (all $P$-values $\leq 0.005$ ). For all measured parameters, there was a significant difference between normal tissue and tumour and between cystic $\mathrm{BPH}$ and normal TZ (all $P \leq 0.005)$.

ADC was able to distinguish tumour from benign tissue with a sensitivity and specificity of $92.3 \%$ and $100 \%$ in the gland TZ (outlines) and right mid PZ posteriorly (arrows). Both lesions positive on $\mathrm{K}_{\text {app }}$ and MTR maps; note clear zonal differentiation seen on MTR maps. Target biopsy, PZ lesion: Gleason $3+4$ in $2 / 2$ cores $50 \%$, up to $8 \mathrm{~mm}$; TZ lesion: Gleason $3+3$ in $2 / 2$ cores $50 \%$, up to $4 \mathrm{~mm}$

$\mathrm{PZ}$ and $100 \%$ and $100 \%$ in the $\mathrm{TZ}$, respectively. $\mathrm{K}_{\text {app }}$ differentiated tumour in the $\mathrm{PZ}$ with $76.9 \%$ sensitivity and $83.3 \%$ specificity and in the TZ with $69.2 \%$ sensitivity and $100 \%$ specificity. MTR distinguished tumour from benign tissue with sensitivity and specificity of $76.9 \%$ and $86.7 \%$, respectively, in the $\mathrm{PZ}$ and $76.9 \%$ and $76.7 \%$, respectively, in the $\mathrm{TZ}$ (Figs. 2, 3 and 4). The ROC area-under-the-curve (AUC) values from the diagnostic metrics ranged from 0.767 to 1.000 for separating tumour from benign tissue (Table 3, Fig. 5). A comparison of AUCs using the bootstrapping method showed no statistically significant difference between $\mathrm{ADC}$ and $\mathrm{D}_{\text {app }}$ in the $\mathrm{TZ}$ or PZ $(p=1.0$ and 0.670 ,
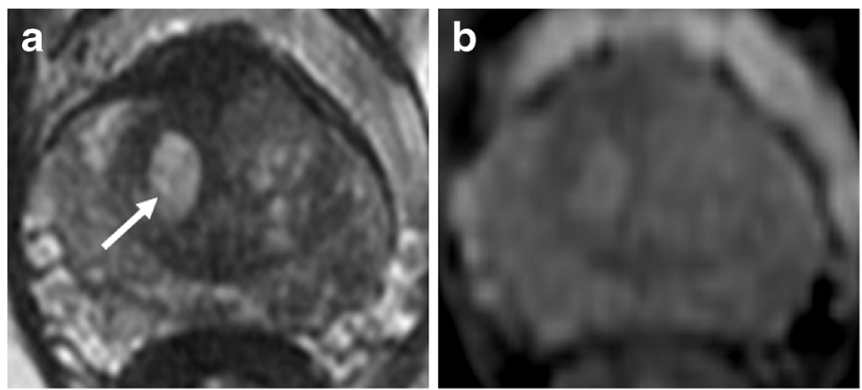

Fig. 4. Cystic BPH demonstrated by magnetisation transfer imaging. A $67-$ year-old patient, PSA $6.39 \mathrm{ng} / \mathrm{ml}$; target biopsy shows Gleason $3+4$ tumour in the left mid TZ (not shown). T2-weighted imaging (a) shows a

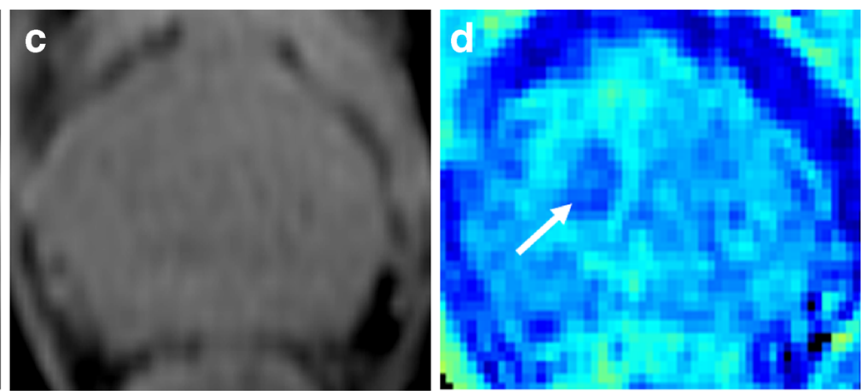

cystic area of BPH in the right apex transition zone (arrow); the area appears as high signal with MT "on" imaging (b), isointense on MT "off" imaging (c), and low signal on the magnetization transfer ratio image (d) 
Table 3. Diagnostic utility of each respective metric in separating normal tissue vs. tumour

\begin{tabular}{|c|c|c|c|c|}
\hline & Cut-off & AUC & Sensitivity (\%) & Specificity (\%) \\
\hline \multicolumn{5}{|l|}{$A D C \times 10^{-3} \mathrm{~mm}^{2} \mathrm{~s}^{-1}$} \\
\hline Transition zone & 1.076 & 1.000 & 100.0 & 100.0 \\
\hline Peripheral zone & 1.037 & 0.979 & 92.3 & 100.0 \\
\hline \multicolumn{5}{|l|}{$D_{a p p} \times 10^{-3} \mathrm{~mm}^{2} \mathrm{~s}^{-1}$} \\
\hline Transition zone & 1.524 & 1.000 & 100.0 & 100.0 \\
\hline Peripheral zone & 1.481 & 0.990 & 100.0 & 96.7 \\
\hline \multicolumn{5}{|l|}{$K_{a p p}$ (unitless) } \\
\hline Transition zone & 0.820 & 0.772 & 69.2 & 100.0 \\
\hline Peripheral zone & 0.621 & 0.772 & 76.9 & 83.3 \\
\hline \multicolumn{5}{|l|}{ MTR (\%) } \\
\hline Transition zone & 28.2 & 0.767 & 76.9 & 76.7 \\
\hline Peripheral zone & 24.0 & 0.828 & 76.9 & 86.7 \\
\hline
\end{tabular}

AUC, area under the curve

respectively). However, the diagnostic utility measured using AUCs was significantly better for ADC compared to $\mathrm{K}_{\text {app }}$ and MTR in both the TZ ( $p=0.036$ and 0.007$)$ and PZ $(p=0.028$ and 0.014).

The ability of the parameters to distinguish low- (Gleason $3+3$ ) from high-grade (Gleason $\geq 3+4$ ) was also assessed. A separate analysis was performed for $\mathrm{PZ}$ and $\mathrm{TZ}$ because of the different values derived for normal tissue for all metrics. The Gleason $3+3$ group included four PZ and four TZ tumours; the GS $\geq 3+4$ included nine PZ and nine TZ tumours. All measured parameters were poor at separating low- and high-grade disease for both PZ $(p=0.414-0.825)$ and TZ $(p=0.148$ 0.825) (Table 4).
ADC showed a strong overall correlation to $\mathrm{D}_{\text {app }}(r=-$ 0.862 ), as expected. ADC showed a moderate inverse correlation to MTR ( $r=-0.618)$ and a good inverse correlation to $\mathrm{K}_{\text {app }}(r=-0.767)$ in normal tissue, with a lower correlation in tumour tissue ( $r=-0.459$ and -0.444 , respectively) (Table 5). MTR showed a moderate correlation to both $\mathrm{K}_{\text {app }}(r=0.570)$ and $\mathrm{D}_{\text {app }}(r=-0.537)$ in normal tissue, but conversely showed a notably poor correlation to $\mathrm{K}_{\text {app }}(r=0.141)$ and $\mathrm{D}_{\text {app }}(r=-$ 0.024 ) in tumour tissue.

\section{Discussion}

In this study we assessed the potential added value of magnetisation transfer imaging and non-Gaussian diffusion kurtosis imaging to conventional mpMRI sequences for the detection of prostate cancer in a re-biopsy population, using targeted transperineal biopsy as the reference standard. All four measured parameters were able to distinguish benign from tumour tissue, but performed poorly at differentiating low- (GS 3+3) from high-grade $(\geq 3+4)$ disease. Standard diffusion-weighted imaging ADC maps showed a moderate overall inverse correlation with both $\mathrm{K}_{\mathrm{app}}$ and MTR, but there was no observable correlation between MTR maps and DKI parameters within tumours.

A number of previous studies have looked at the ability of DKI to differentiate tumour grade compared to standard diffusion-weighted imaging with mono-exponential modelling. DKI parameters have repeatedly been shown to distinguish benign from tumour tissue, with several studies suggesting that the kurtosis metric K outperforms ADC for differentiating low- and high-grade tumours [10, 11, 25-27]. a

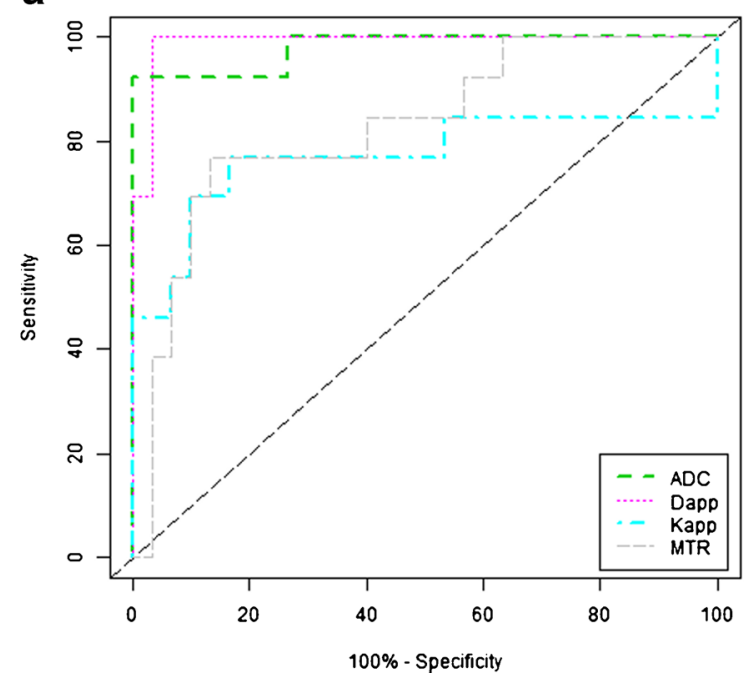

b

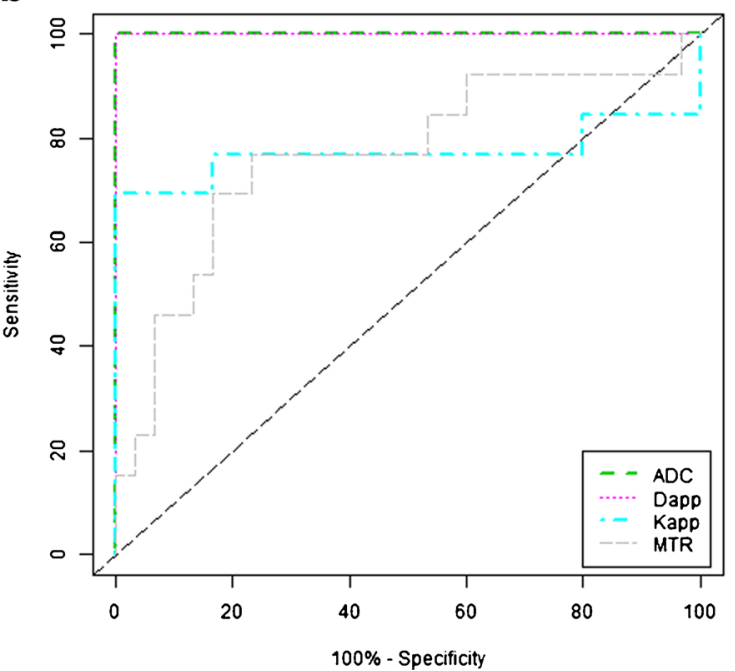

Fig. 5. Receiver-operating characteristic curves of the performance of $A D C, D_{a p p}, K_{a p p}$, and MTR for differentiating benign tissue and tumour in the peripheral (a) and transition zone (b) 
Table 4. Ability of metrics to distinguish low- (Gleason 3+3) from high-grade (Gleason $\geq 3+4$ ) tumours

\begin{tabular}{lccc}
\hline & Gleason $3+3$ & Gleason $\geq 3+4$ & $p$-value \\
\hline Peripheral zone & $n=4$ & $n=9$ & \\
$\mathrm{ADC} \times 10^{-3} \mathrm{~mm}^{2} \mathrm{~s}^{-1}$ & $0.905[0.860-0.949]$ & $0.902[0.893-0.1036]$ & 0.711 \\
$D_{\text {app }} \times 10^{-3} \mathrm{~mm}^{2} \mathrm{~s}^{-1}$ & $1.275[1.047-1.463]$ & $1.167[0.740-1.196]$ & 0.414 \\
$K_{\text {app }}($ unitless $)$ & $0.759[0.643-0.902]$ & $0.716[0.480-0.867]$ & 0.711 \\
MTR $(\%)$ & $26.1[23.4-28.4]$ & $25.0[24.0-27.5]$ & 0.825 \\
Transition zone & $n=4$ & $n=9$ & \\
ADC $\times 10^{-3} \mathrm{~mm}^{2} \mathrm{~s}^{-1}$ & $0.881[0.864-0.887]$ & $0.830[0.799-0.884]$ & 0.414 \\
$\mathrm{D}_{\text {app }} \times 10^{-3} \mathrm{~mm}^{2} \mathrm{~s}^{-1}$ & $0.926[0.889-0.979]$ & $1.006[0.745-1.083]$ & 0.825 \\
$\mathrm{~K}_{\text {app }}($ unitless $)$ & $0.695[0.508-0.924]$ & $0.975[0.820-1.035]$ & 0.414 \\
MTR $(\%)$ & $31.9[31.3-32.2]$ & $29.6[26.3-31.5]$ & 0.148 \\
\hline
\end{tabular}

Mean values listed, interquartile range in parentheses
However, these studies also note a strong inverse correlation between $\mathrm{K}$ with $\mathrm{ADC}$, as supported by our data, raising the question of the additional clinical benefit over existing DWI sequences, given the increased technical complexity of DKI in terms of post-processing and interpretation. Indeed, more recent work and a large retrospective study using prostatectomy as a reference standard suggested no additional benefit of DKI sequences over conventional DWI $[12,28]$. A possible explanation is the use of a clinically derived ADC map in these studies (using a high b-value of 1000) in contrast to earlier work where the ADC map was derived from the DKI sequences with high b-values ranging from 1400-2000 s/mm². Current guidelines caution against using such high b-values for ADC calculation because of the non-mono-exponential decay and concerns about a reduced signal-to-noise ratio and noise-floor bias [8]; this may therefore have affected the performance of the ADC measurements in these studies. We also derived ADC maps from standard clinical DWI sequences, which may explain the lack of added benefit of DKI parameters. Another explanation may be the longer echo time (TE) used in the DKI sequence to achieve the diffusion weighting, which reduced the intrinsic SNR of the $D_{\text {app }}$ obtained using lower b-values, as suggested by Roethke et al. [28].

Two previous studies have investigated the utility of MTI in prostate cancer $[17,18]$. Both were performed at $1.5 \mathrm{~T}$ and assessed only PZ tumours, used systematic TRUS biopsy as the reference standard, and did not differentiate between Gleason grades. Our results support the findings of these studies that normal TZ and PZ tumours have a higher MTR than normal PZ. The difference in MTR between normal PZ and normal $\mathrm{TZ}$ is expected given the difference in composition between the tissue types: the lower value in the PZ may be explained by a relatively loose stroma, a larger extracellular space, and a fluid-filled glandular cavity [29]. The mean MTR values derived here are comparable to those in the study by Arima et al. [18] for both normal TZ (26.9\% vs. 25.5\%) and PZ tumour (25.0\% vs. $30.6 \%$ ), but higher than the study by Kumar et al. (7.01\% and $8.29 \%$, respectively) [17]. Conversely our results for normal PZ (20.0\%) were much higher than these two studies $(8.0 \%$ and $6.15 \%$, respectively). This discrepancy may relate to the fact that MTR is an arbitrary measure and depends on the characteristics of the pulse sequence; the Kumar et al. study differed from ours in being a 2D sequence with a long TR. In addition, the earlier studies may have effects relating to residual post-biopsy haemorrhage, which will predominantly affect the $\mathrm{PZ}$ rather than the TZ when systematic TRUS biopsy is performed, but will typically be excluded from areas containing cancer [30]; thus only normal $\mathrm{PZ}$ rather than $\mathrm{TZ}$ or $\mathrm{PZ}$ tumour will be affected.

A number of previous studies have shown ADC to decrease as tumour Gleason grade increases, albeit with a degree of overlap in values between tumour grades [31-33].
Table 5. Correlation between assessed metrics (Pearson's Rho)

\begin{tabular}{llll}
\hline Comparators & $\begin{array}{l}\text { Tumour Tissue }(\mathrm{TZ}+ \\
\mathrm{PZ})\end{array}$ & $\begin{array}{l}\text { Normal Tissue }(\mathrm{TZ}+ \\
\mathrm{PZ})\end{array}$ & $\begin{array}{l}\text { Normal }+ \text { Tumour Tissue }(\mathrm{TZ}+ \\
\mathrm{PZ}\end{array}$ \\
\hline ADC vs Kapp & $\mathbf{- 0 . 4 4 4}$ & $\mathbf{- 0 . 7 6 7}$ & $\mathbf{- 0 . 6 4 1}$ \\
ADC vs D & -0.041 & -0.790 & 0.862 \\
ADC vs MTR & $\mathbf{- 0 . 4 5 9}$ & $\mathbf{- 0 . 6 1 8}$ & $\mathbf{- 0 . 6 3 3}$ \\
$\mathrm{K}_{\text {app }}$ vs D & 0.646 & -0.532 & -0.359 \\
$\mathbf{K}_{\text {app }}$ vs MTR & $\mathbf{0 . 1 4 1}$ & $\mathbf{0 . 5 7 0}$ & $\mathbf{0 . 4 2 9}$ \\
$\mathrm{D}_{\text {app }}$ vs MTR & -0.024 & -0.537 & -0.531 \\
\hline
\end{tabular}


Although there was a trend for lower ADC values in highergrade (Gleason $\geq 3+4$ ) tumours in our cohort, this was nonsignificant, which may relate to the relatively small sample size, particularly within the PZ. However, the concordance between ADC and Gleason grade has recently been questioned [34], with the demonstration that Gleason 3+3 tumours can have low ADC values [35]. This highlights the need for further work in the area to both improve the understanding of ADC metrics in this regard and assess additional better functional sequences for characterisation of tumour aggressiveness. MTR showed a moderately strong correlation to both $\mathrm{K}_{\text {app }}(r=0.57)$ and $\mathrm{D}_{\text {app }}(r=-0.537)$ in normal tissue, yet no correlation in tumour tissue $(r=0.141$ and -0.024 , respectively). Of note, there was a non-significant trend for increasing $\mathrm{K}_{\text {app}}$, but conversely lower MTR in higher-grade transition zone tumours. These divergent results combined with the poor correlation of MTI and DKI suggest that the techniques offer different but complementary information on the tumour microenvironment. Interestingly, both tumour $\mathrm{K}_{\text {app }}$ and MTR showed a high inverse correlation to ADC $(r=-0.444$ and 0.459 , respectively), suggesting that cellularity is partly contributing to these metrics, whilst other factors are contributing to their divergent values. At higher Gleason grades, the glandular structure of the prostate is progressively disrupted with increased cellularity and a reduction in the stromal matrix and luminal space [21]. This increased heterogeneity can therefore help to explain a trend for higher $\mathrm{K}_{\mathrm{app}}$ values observed in higher-grade tumours [10]. Higher-grade prostate cancer is expected to result in an increased number of intracellular bound water molecules due to increased cellular density [36]; however, this is counteracted by a breakdown of the normal extensive extracellular matrix [37]. These competing processes may provide an explanation for the divergence among DWI, DKI, and MTI demonstrated here in tumours.

Our study has a number of limitations. The numbers within the cohort were relatively low; this may have particularly affected attempts to differentiate high- and low-grade tumours, where further sub-division became necessary. Targeted biopsy ensured that tissue was sampled from the outlined lesion; however, as with any biopsy technique, this is prone to sampling error and may misclassify the grade compared to the more robust gold standard of prostatectomy [38]. Targets were prospectively chosen based on clinical mpMRI sequences, and DKI/MTR values were not used to inform the biopsy decision. This may have led to a bias towards lesions with restricted diffusion and low ADC, particularly within the PZ where this is considered the key diagnostic sequence. Conversely, there may be a bias against areas that demonstrated changes on DKI or MTR but not on conventional MRI sequences as they would not have been biopsied.

In conclusion, ADC, MTI, and DKI readily distinguished benign tissue from tumour, but none of the measured parameters reliably differentiated low- from high-grade disease.
Differences between DKI and MTI at higher Gleason grades may be explained by changes in the cellularity, stromal matrix, and luminal space. DKI and MTI may therefore offer different but potentially complementary information on the tumour microenvironment.

Acknowledgements The authors acknowledge research support from Cancer Research UK, National Institute of Health Research Cambridge Biomedical Research Centre, Cancer Research UK and the Engineering and Physical Sciences Research Council Imaging Centre in Cambridge and Manchester and the Cambridge Experimental Cancer Medicine Centre.

Funding The authors state that this work has not received any funding.

\section{Compliance with ethical standards}

Guarantor The scientific guarantor of this publication is Tristan Barrett.

Conflict of interest The authors of this manuscript declare no relationships with any companies, whose products or services may be related to the subject matter of the article.

Statistics and biometry One of the authors has significant statistical expertise (AJP).

No complex statistical methods were necessary for this paper.

Ethical approval Institutional Review Board approval was obtained.

Informed consent Written informed consent was obtained from all subjects (patients) in this study.

\section{Methodology \\ - prospective \\ - diagnostic or prognostic study \\ - performed at one institution}

Open Access This article is distributed under the terms of the Creative Commons Attribution 4.0 International License (http:// creativecommons.org/licenses/by/4.0/), which permits unrestricted use, distribution, and reproduction in any medium, provided you give appropriate credit to the original author(s) and the source, provide a link to the Creative Commons license, and indicate if changes were made.

\section{References}

1. Siegel RL, Miller KD, Jemal A (2017) Cancer Statistics, 2017. CA Cancer J Clin. 67:7-30

2. Lecornet E, Ahmed HU, Hu Yet al (2012) The accuracy of different biopsy strategies for the detection of clinically important prostate cancer: a computer simulation. J Urol. 188:974-980

3. Kvåle R, Møller B, Wahlqvist R et al (2009) Concordance between Gleason scores of needle biopsies and radical prostatectomy specimens: a population-based study. BJU Int. 103:1647-1654

4. National Institute for Health and Care Excellence (2014) Prostate cancer: diagnosis and treatment. Clinical guideline 175 (Update of clinical guideline 58): https://www.nice.org.uk/guidance/cg175. Accessed 10/5/2017

5. Ahmed HU, El-Shater Bosaily A, Brown LC et al (2017) Diagnostic accuracy of multi-parametric MRI and TRUS biopsy 
in prostate cancer (PROMIS): a paired validating confirmatory study. Lancet. 89:815-822

6. Woo S, Suh CH, Kim SY, Cho JY, Kim SH (2017) Diagnostic performance of Prostate Imaging Reporting and Data System Version 2 for Detection of Prostate Cancer: A systematic review and diagnostic meta-analysis. Eur Urol. pii S0302-2838:3006730062

7. Turkbey B, Albert PS, Kurdziel K, Choyke PL (2009) Imaging localized prostate cancer: current approaches and new developments. AJR Am J Roentgenol. 192:1471-1480

8. Barrett T, Turkbey B, Choyke PL (2015) PI-RADS version 2: what you need to know. Clin Radiol. 70:1165-1176

9. Jensen JH, Helpern JA, Ramani A, Lu H, Kaczynski K (2005) Diffusional kurtosis imaging: the quantification of non-Gaussian water diffusion by means of magnetic resonance imaging. Magn Reson Med. 53:1432-1440

10. Lawrence EM, Warren AY, Priest AN et al (2016) Evaluating prostate cancer using fractional tissue composition of radical prostatectomy specimens and pre-operative diffusional kurtosis magnetic resonance imaging. PLoS One. 11:e0159652

11. Wang Q, Li H, Yan X, Wu CJ, Liu XS, Shi HB, Zhang YD (2015) Histogram analysis of diffusion kurtosis magnetic resonance imaging in differentiation of pathologic Gleason grade of prostate cancer. Urol Oncol. 33:337e15-337e24

12. Tamada T, Prabhu V, Li J, Babb JS, Taneja SS, Rosenkrantz AB (2017) Prostate cancer: Diffusion-weighted MR imaging for detection and assessment of aggressiveness-comparison between conventional and kurtosis models. Radiology. 284:100-108

13. Henkelman RM, Stanisz GJ, Graham SJ (2001) Magnetization transfer in MRI: A review. NMR Biomed. 14:57-64

14. Wolff SD, Balaban RS (1994) Magnetization transfer imaging: practical aspects and clinical applications. Radiology 192:593-599

15. Kurki T, Lundbom N, Kalimo H, Valtonen S (1995) MR classification of brain gliomas: value of magnetization transfer and conventional imaging. Magn Reson Imaging 13:501-511

16. Garcia M, Gloor M, Bieri O et al (2015) Imaging of primary brain tumors and metastases with fast quantitative 3-dimensional magnetization transfer. J Neuroimaging. 25:1007-1014

17. Kumar V, Jagannathan NR, Kumar R, Thulkar S, Gupta SD, Hemal AK, Gupta NP (2008) Evaluation of the role of magnetization transfer imaging in prostate: a preliminary study. Magn Reson Imaging. 26:644-649

18. Arima K, Hayashi N, Yanagawa M, Kawamura J, Kobayashi S, Takeda K, Sugimura Y (1999) The progress in diagnostic imaging for staging of bladder and prostate cancer: endorectal magnetic resonance imaging and magnetization transfer contrast. Hinyokika Kiyo. 45:553-557

19. Barentsz JO, Richenberg J, Clements R, European Society of Urogenital Radiology et al (2012) ESUR prostate MR guidelines 2012. Eur Radiol. 22:746-757

20. Rosenkrantz AB, Kim S, Lim RP et al (2013) Prostate cancer localization using multiparametric MR imaging: comparison of Prostate Imaging Reporting and Data System (PI-RADS) and Likert scales. Radiology. 269:482-492

21. Lawrence EM, Tang SY, Barrett T et al (2014) Prostate cancer: performance characteristics of combined $\mathrm{T}_{2} \mathrm{~W}$ and DW-MRI scoring in the setting of template transperineal re-biopsy using MRTRUS fusion. Eur Radiol. 24:1497-1505

22. Röthke M, Blondin D, Schlemmer HP, Franiel T (2013) PI-RADS classification: structured reporting for MRI of the prostate. Rofo. $185: 253-261$
23. Kuru TH, Wadhwa K, Chang RT et al (2013) Definitions of terms, processes and a minimum dataset for transperineal prostate biopsies: a standardization approach of the Ginsburg Study Group for Enhanced Prostate Diagnostics. BJU Int. 112:568-577

24. Epstein JL, Allsbrook WC Jr, Amin MB, ISUP Grading Committee et al (2005) The 2005 international society of urological pathology (ISUP) consensus conference on Gleason grading of prostatic carcinoma. Am J Surg Path 29:1228-1242

25. Rosenkrantz AB, Sigmund EE, Johnson G et al (2012) Prostate cancer: feasibility and preliminary experience of a diffusional kurtosis model for detection and assessment of aggressiveness of peripheral zone cancer. Radiology. 264:126-135

26. Suo S, Chen X, Wu L et al (2014) Non-Gaussian water diffusion kurtosis imaging of prostate cancer. Magn Reson Imaging 32:421427

27. Tamura C, Shinmoto H, Soga S et al (2014) Diffusion kurtosis imaging study of prostate cancer: preliminary findings. J Magn Reson Imaging. 40:723-729

28. Roethke MC, Kuder TA, Kuru TH et al (2015) Evaluation of diffusion kurtosis imaging versus standard diffusion imaging for detection and grading of peripheral zone prostate cancer. Invest Radiol. 50:483-489

29. Wang XZ, Wang B, Gao ZQ et al (2009) Diffusion-weighted imaging of prostate cancer: correlation between apparent diffusion coefficient values and tumor proliferation. J Magn Reson Imaging 29:1360-1366

30. Barrett T, Vargas HA, Akin O, Goldman DA, Hricak H (2012) Value of the hemorrhage exclusion sign on T1-weighted prostate MR images for the detection of prostate cancer. Radiology. 263: 751-757

31. Hambrock T, Somford DM, Huisman HJ et al (2011) Relationship between apparent diffusion coefficients at 3.0-T MR imaging and Gleason grade in peripheral zone prostate cancer. Radiology. 259: 453-461

32. Barrett T, Priest AN, Lawrence EM et al (2015) Ratio of tumor to normal prostate tissue apparent diffusion coefficient as a method for quantifying DWI of the prostate. AJR Am J Roentgenol. 205: W585-W593

33. Donati OF, Mazaheri Y, Afaq A et al (2014) Prostate cancer aggressiveness: assessment with whole-lesion histogram analysis of the apparent diffusion coefficient. Radiology. 271:143-145

34. Chatterjee A, Watson G, Myint E, Sved P, McEntee M, Bourne R (2015) Changes in epithelium, stroma, and lumen space correlate more strongly with Gleason pattern and are stronger predictors of prostate ADC changes than cellularity metrics. Radiology. 277: $751-762$

35. Helfrich O, Puech P, Betrouni N et al (2017) Quantified analysis of histological components and architectural patterns of Gleason grades in apparent diffusion coefficient restricted areas upon diffusion weighted MRI for peripheral or transition zone cancer locations. J Magn Reson Imaging. https://doi.org/10.1002/jmri.25716

36. Jia G, Abaza R, Williams JD et al (2011) Amide proton transfer MR imaging of prostate cancer: a preliminary study. J Magn Reson Imaging. 33:647-654

37. Takayama Y, Nishie A, Sugimoto M et al (2016) Amide proton transfer (APT) magnetic resonance imaging of prostate cancer: comparison with Gleason scores. MAGMA 29:671-679

38. Lanz C, Cornud F, Beuvon F et al (2016) Gleason score determination with transrectal ultrasound-magnetic resonance imaging fusion guided prostate biopsies - are we gaining in accuracy? J Urol. 195:88-93 